\title{
Incidence of Aneurysmal Subarachnoid Hemorrhage with Procedures Requiring General Anesthesia in Patients with Unruptured Intracranial Aneurysms
}

\author{
Hesham Masoud $^{a}$ Vijaylakshmi Nair ${ }^{a} \quad$ Adekorewale Odulate-Williams $^{\text {a }}$ \\ Sameer Sharma ${ }^{a}$ Grahame Gould $^{a}$ Joshua Thatcher ${ }^{b}$ \\ Thanh N. Nguyen ${ }^{b}$ \\ aSUNY Upstate Medical University, Upstate University Hospital, Syracuse, NY, USA; \\ boston University School of Medicine, Boston Medical Center, Boston, MA, USA
}

\section{Keywords}

Subarachnoid hemorrhage · Unruptured intracranial aneurysms · Anesthesia · Cerebral aneurysm $\cdot$ Rupture $\cdot$ Intubation

\begin{abstract}
Background: The role of general anesthesia in precipitating aneurysm rupture is not clearly defined. In this study, we aimed to assess the natural history of unruptured aneurysms in patients undergoing non-aneurysm-related procedures requiring general anesthesia. Methods: Retrospective review of consecutive patients with untreated intracranial aneurysms that underwent unrelated surgery with operative note documentation of general anesthesia. Events of intraoperative and postoperative subarachnoid hemorrhage were recorded to determine the incidence of rupture. Results: A total of 110 patients harboring 134 unsecured aneurysms were studied. The mean age was 56.5 years (range, 17-92), and 68\% were women ( $n=75 / 110)$. Mean aneurysm size was $3.5 \mathrm{~mm}$ (range 1.5-17). A total of 208 procedures were performed under general anesthesia. There were no events of subarachnoid hemorrhage in 5.7 years of follow-up. Conclusion: In our study, general anesthesia did not precipitate aneurysm rupture, and there were no instances of subarachnoid hemorrhage during the follow-up period. Our results suggest a benign natural history for aneurysms undergoing unrelated general anesthesia. However, this should be interpreted with caution given limitations related to our small sample size and retrospective study design.




\section{Introduction}

Advances in neuroimaging and an increased reliance on its use in clinical practice has led to an increase in the discovery of patients harboring incidental cerebral aneurysms. The overall prevalence of unruptured intracranial aneurysms (UIAs) is estimated to be $3 \%$ [1].

The most feared complication is subarachnoid hemorrhage (SAH) from an aneurysm rupture. Fortunately, the majority of aneurysms do not, and may never manifest clinically during a patient's lifetime. The incidence of SAH from an unruptured aneurysm is approximately 10 per 100,000 people per year [2]. Although rare, aneurysmal SAH is associated with a high morbidity and mortality [3]. Epidemiological studies have reported mortality rates ranging from 8 to $67 \%$ [4].

The estimated average annual incidence of rupture has been reported to be low; the prospective phase of the International Study of Unruptured Intracranial Aneurysms (ISUIA) reported an overall yearly rupture risk of $0.7 \%$ [5], and more recently Juvela et al. [6] published an approximate annual incidence of 1.1\%. In a Japanese cohort of 5,720 patients with UIAs, an annual rupture rate of $0.95 \%$ was reported. Risk of rupture increased with size of the aneurysm and the presence of a daughter sac on the aneurysm fundus wall [7]. Risk of SAH is largely dependent on aneurysm size, location, and history of previous SAH [5]; however, other factors have also been associated with an elevated risk of aneurysm rupture, including smoking status and female sex [8].

Patients with ruptured and unruptured aneurysms are routinely treated under general anesthesia (GA) without incident, but there are limited data available regarding its safety in patients undergoing procedures where the goal of surgery is not related to treatment of the aneurysm. There is concern that the induction of GA, intubation, or extubation may have the potential to alter patient hemodynamics, leading to aneurysm rupture [9].

The purpose of our study is to assess the natural history of aneurysm rupture in patients undergoing non-aneurysm-related procedures requiring GA.

\section{Methods}

This was a retrospective double center study approved by the local institutional review boards.

\section{Patient Selection}

Patients were identified from the hospital databases of 2 tertiary academic medical centers for patients via a search of the ICD-9 code for cerebral aneurysm (437.3) from January 2000 to February 2013 from Boston Medical Center, and from Upstate University Hospital using ICD-9 code for cerebral aneurysm (437.3) from January 2000 to September 30th 2015 and ICD-10 code (167.1) from October 2015 to April 2016. Patients were selected if they had undergone a non-cerebrovascular procedure requiring GA while harboring an unruptured, unsecured intracranial aneurysm. We also included a subgroup of women who delivered via cesarean section under spinal anesthesia.

Patients with a simultaneous diagnosis of SAH (ICD-9 code 430) and history of known ruptured aneurysm in their medical history were excluded. We also excluded patients without available imaging reports to confirm aneurysm presence and those patients who underwent procedures without documentation of GA. Patients with procedures performed using local anesthesia or conscious sedation were excluded, with the rationale that minor procedures not requiring GA did not pose a direct risk of aneurysm rupture.

Aneurysm Identification, Size, and Location

The medical record was reviewed to confirm the presence and number of unruptured cerebral aneurysms. Aneurysm locations and sizes were gathered from the neuroimaging reports and verified by review of available imaging studies. 
Masoud et al.: General Anesthesia and Aneurysmal SAH

Table 1. Aneurysm characteristics

\begin{tabular}{lcl}
\hline Location & $\begin{array}{l}\text { Aneurysms, } \\
n\end{array}$ & $\begin{array}{l}\text { Mean size } \\
\text { (range), mm }\end{array}$ \\
\hline Anterior circulation aneurysms $(n=114 / 134)$ & \\
$\quad$ Cavernous-ICA & 20 & $3.5(2-8.8)$ \\
Paraophthalmic-ICA & 40 & $3.5(1.5-10)$ \\
Carotid-terminus & 8 & $3.3(2-6)$ \\
Anterior choroidal & 2 & $6.3(2.5-10)$ \\
MCA & 24 & $3.7(1.5-16)$ \\
Acomm & 20 & $3.8(2-6)$ \\
Posterior circulation aneurysms $(n=20 / 134)$ & \\
Pcomm & 13 & $2.9(1.5-5)$ \\
PCA & 2 & $3.4(2-4.8)$ \\
Basilar & 2 & $4.5(2-7)$ \\
SCA & 1 & 2.0 \\
PICA & 2 & 2.0 \\
\hline
\end{tabular}

ICA, internal carotid artery; Acomm, anterior communicating artery; MCA, middle cerebral artery; Pcomm, posterior communicating artery; PCA, posterior cerebral artery; SCA, superior cerebellar artery; PICA, posterior inferior cerebellar artery.

Identification of Patients Who Had Undergone a Procedure and Follow-Up

A search of the medical record was conducted for documentation of an operative note with any report of anesthesia. The date of the procedure was cross-checked with date of aneurysm diagnosis. Patients were followed for a minimum of 1 week after procedure and up to the end of the study period.

Primary Outcome

The primary outcome was SAH within 7 days after an unrelated procedure requiring GA. The electronic medical record hospital discharge summaries were reviewed to capture any potential missed cases of SAH attributed to procedure-related aneurysm rupture. For aneurysms that were clipped or coiled during the study period, follow-up time was calculated as time from aneurysm discovery to the date of treatment.

\section{Results}

Our study of patients from 2 participating centers included a total of 110 patients harboring 134 unsecured aneurysms. The mean age of our cohort was 57 years (range, 17-92), and 68\% were women $(n=75 / 110)$. The mean aneurysm size was $3.5 \mathrm{~mm}$ (range, $1.5-17)$. The majority of aneurysms studied were located in the anterior circulation $(85 \%, n=114 / 134)$; specific locations and sizes are listed in Table 1 . Five aneurysms were treated during the study period ( 2 with coiling, and 3 with clipping). There were 208 procedures performed under GA listed in Table 2. There were no events of SAH captured in 5.7 years of follow-up.

\section{Discussion}

In clinical neurointerventional practice, we are routinely asked to comment on the risk of aneurysm rupture when there is a potential treatment intervention that may augment risk of aneurysm rupture, such as initiating anticoagulation [10]. Another common scenario is a patient with a known UIA with plans for future surgery unrelated to the aneurysm. While the risk of rupture in this situation is likely low and more dependent on factors intrinsic to the 


\begin{tabular}{l|l}
\hline DOI: $10.1159 / 000490582$ & $\begin{array}{l}\text { C 2018 S. Karger AG, Basel } \\
\text { www.karger.com/ine }\end{array}$ \\
\hline
\end{tabular}

Masoud et al.: General Anesthesia and Aneurysmal SAH

Table 2. Procedures performed under general anesthesia

\begin{tabular}{lc}
\hline Type of surgery & Procedures, $n$ \\
\hline Orthopedic & 60 \\
General & 35 \\
ENT & 24 \\
Vascular & 15 \\
Spine & 16 \\
Urology & 13 \\
Cardiothoracic & 10 \\
Plastic & 8 \\
OBGYN & 7 \\
Neurosurgery & 6 \\
Ophthalmology & 4 \\
Oncology & 3 \\
Colorectal & 3 \\
OMF & 2 \\
Podiatry & 1 \\
Imaging & 1 \\
\hline Total & 208 \\
\hline
\end{tabular}

ENT, ear nose and throat; OBGYN, obstetrics and gynecology; OMF, oral and maxillofacial.

aneurysm [11], the role of unrelated surgery in precipitating rupture is uncertain. There is limited literature available to guide the practitioner in assessing risk. Tsementzis and Hitchcock [9] reported a study of 404 patients with ruptured intracerebral aneurysms treated with microsurgical clipping, $2 \%$ experienced re-rupture at the time of induction and intubation. Seven out of the eight reported ruptures were noted to have had complicated intubations or coughed during induction, suggesting that airway manipulation and a subsequent elevation in blood pressure, may have precipitated the aneurysm ruptures.

Common strategies to reduce the risk of aneurysm rupture during anesthesia induction include careful monitoring and control of the patient's blood pressure, and avoiding spikes of blood pressure over 140 or $150 \mathrm{~mm}$ Hg systolic. Control of nausea and vomiting, minimizing coughing may further mitigate risk.

The recognition of intraoperative rupture (IOR) can be challenging. Neurophysiological monitoring is helpful in this regard. A gradual increase in blood pressure accompanied by a precipitous drop in heart rate may signal an IOR. Changes in the neurological exam may be difficult to assess given GA; however, raised intracranial pressure and herniation can be recognized clinically with a unilateral change in pupil size [12]. The anesthetic care of IOR is complex, and aneurysm rupture in a closed skull carries a worse prognosis due to elevation in intracranial pressure impairing cerebral perfusion [13].

In our study, none of the 134 aneurysms of 110 patients ruptured during our follow-up period with a mean of 5.7 patient years of follow-up. A total of 208 procedures were uneventful, and there were no instances of SAH in the immediate postoperative period. The majority of patients studied $(n=85 / 110)$ came from a single center; in this cohort, aneurysm multiplicity was noted in $25 \%(n=21 / 85)$. There was a family history of cerebral aneurysms in $35 \%$ $(n=30 / 85)$. These patients also carried diagnoses associated with elevated risk [14], including hypertension (71\%, $\mathrm{n}=60 / 85)$, active tobacco smoking ( $54 \%, n=46 / 85)$, or both $(46 \%, n=$ $39 / 85)$. Despite this high-risk subgroup, there were no instances of periprocedural SAH.

Limitations in our study include the retrospective design and small sample size. The majority of UIAs followed arose from the anterior circulation and measured less than $10 \mathrm{~mm}$. 
It is known that these smaller aneurysms are associated with the lowest rates of rupture [5]. In our cohort, the mean aneurysm size was $3.5 \mathrm{~mm}$, which may further explain the absence of ruptures in our study.

It is possible that a number of UIAs with elevated risk of SAH were preemptively treated prior to planned surgery, thereby introducing a selection bias. As such, we cannot exclude the possibility of a type I error, and it is important to note that intraprocedural aneurysm rupture may be challenging to identify due to patient sedation, making management more difficult.

\section{Conclusion}

In this study, non-cerebral surgical procedures performed under GA in patients harboring unruptured intracerebral aneurysms did not precipitate rupture during or within 1 week of the surgery. Our study suggests that unrelated surgery is likely safe, but this subgroup of aneurysm patients requires further investigation to substantiate our conclusions with larger prospective multicenter studies.

\section{Disclosure Statement}

All listed authors disclose no conflict of interest relevant to this study. The authors received no related sponsorship or funding.

\section{References}

1 Vlak MH, Algra A, Brandenburg R, Rinkel GJ: Prevalence of unruptured intracranial aneurysms, with emphasis on sex, age, comorbidity, country, and time period: a systematic review and meta-analysis. Lancet Neurol 2011; 10:626-636.

2 Mackey J, Brown RD, Moomaw CJ, Sauerbeck L, Hornung R, Gandhi D, et al: Unruptured intracranial aneurysms in the Familial Intracranial Aneurysm and International Study of Unruptured Intracranial Aneurysms cohorts: differences in multiplicity and location. J Neurosurg DOI 10.3171/2012.4.JNS111822.

3 van Gijn J, Rinkel GJ: Subarachnoid haemorrhage: diagnosis, causes and management. Brain 2001;124(Pt 2): 249-278.

4 Nieuwkamp DJ, Setz LE, Algra A, Linn FH, de Rooij NK, Rinkel GJ: Changes in case fatality of aneurysmal subarachnoid haemorrhage over time, according to age, sex, and region: a meta-analysis. Lancet Neurol 2009;8: 635-642.

5 Wiebers DO, Whisnant JP, Huston J 3rd, Meissner I, Brown RD Jr, Piepgras DG, Forbes GS, Thielen K, Nichols D, O’Fallon WM, Peacock J, Jaeger L, Kassell NF, Kongable-Beckman GL, Torner JC; International Study of Unruptured Intracranial Aneurysms Investigators: Unruptured intracranial aneurysms: natural history, clinical outcome, and risks of surgical and endovascular treatment. Lancet 2003;362:103-110.

6 Juvela S, Poussa K, Lehto H, Porras M: Natural history of unruptured intracranial aneurysms. Stroke 2013;44: $2414-2421$.

7 UCAS Japan Investigators, Morita A, Kirino T, Hashi K, Aoki N, Fukuhara S, Hashimoto N, Nakayama T, Sakai M, Teramoto A, Tominari S, Yoshimoto T: The natural course of unruptured cerebral aneurysms in a Japanese cohort. N Engl J Med 2012;366:2474-2482.

8 Korja M, Lehto H, Juvela S: Lifelong rupture risk of intracranial aneurysms depends on risk factors: a prospective Finnish cohort study. Stroke 2014;45:1958-1963.

9 Tsementzis SA, Hitchcock ER: Outcome from "rescue clipping" of ruptured intracranial aneurysms during induction anaesthesia and endotracheal intubation. J Neurol Neurosurg Psychiatry 1985;48:160-163.

10 Tarlov NE, Norbash AN, Nguyen TN: Safety of anticoagulation in patients with unruptured aneurysms. J Neurointerv Surg 2013;5:405-497.

11 Morita A: Current perspectives on the unruptured cerebral aneurysms: origin, natural course, and management. J Nippon Med Sch 2014;81.

12 Chowdhury T, Petropolis A, Wilkinson M, Schaller B, Sandu N, Cappellani RB: Controversies in the anesthetic management of intraoperative rupture of intracranial aneurysm. Anesthesiol Res Pract 2014;2014:595837.

13 Beatty RA: Intraoperative aneurysms rupture during the predissection stage. J Neurol Neurosurg Psychiatry 1990;53:711-712.

14 Jabbarli R, Dinger TF, Darkwah Oppong M, Pierscianek D, Dammann P, Wrede KH, et al: Risk factors for and clinical consequences of multiple intracranial aneurysms: a systematic review and meta-analysis. Stroke 2018; 49:848-855. 